\title{
Nutrient ileal digestibility evaluation of dried mealworm (Tenebrio molitor) larvae compared to three animal protein by-products in growing pigs
}

\author{
J. S. Yoo ${ }^{1, a}$, K. H. Cho ${ }^{1, a}$, J. S. Hong ${ }^{2}$, H. S. Jang ${ }^{3}$, Y. H. Chung ${ }^{3}$, G. T. Kwon ${ }^{4}$, D. G. Shin ${ }^{4}$, and Y. Y. Kim ${ }^{2, *}$
}

* Corresponding Author: Y. Y. Kim
Tel: +82-2-878-5838, Fax: +82-2-878-5839,
E-mail: yooykim@snu.ac.kr

'Daehan feed Co., Ltd., R\&D Center, Incheon 22300, Korea

${ }^{2}$ Department of Agricultural Biotechnology, College of Animal Life Sciences, Seoul National University, Seoul 08826, Korea

${ }^{3}$ Jeonbuk Institute for Food-Bioindustry, Jeonju 54810, Korea

${ }^{4}$ Berry \& Biofood Research Institute, Jeonbuk,

Gochang, 56417, Korea

a These authors contributed equally to this work.

ORCID

J. S. Yoo

https://orcid.org/0000-0002-1995-9858

K. H. Cho

https://orcid.org/0000-0001-5036-3553

J. S. Hong

https://orcid.org/0000-0002-3401-3024

H. S. Jang

https://orcid.org/0000-0002-4294-489X

Y. H. Chung

https://orcid.org/0000-0001-5470-8360

G. T. Kwon

https://orcid.org/0000-0001-8076-4059

D. G. Shin

https://orcid.org/0000-0003-3977-0208

Y. Y. Kim

https://orcid.org/0000-0001-8121-3291

Submitted Aug 30, 2018; Revised Sept 26, 2018: Accepted Oct 29, 2018
Objective: This study was to investigate the nutrient ileal digestibility of dried mealworm (Tenebrio molitor) larvae and compare with those of three animal protein by-products in growing pigs.

Methods: A total of 12 crossbred ([Landrace $\times$ Yorkshire $] \times$ Duroc) growing pigs with average body weights of $24.12 \pm 0.68 \mathrm{~kg}$ were surgically equipped with simple T-cannulas after being deprived of feed for $24 \mathrm{~h}$ according to published surgical procedures. These pigs had a recovery period of two weeks. A total of 12 pigs were assigned to individual metabolic crates and allotted to one of four treatments with 3 replicates in a fully randomized design. Dietary treatments included the following: i) Fish meal, corn-vegetable by-product basal diet $+9.95 \%$ fish meal; ii) Meat meal, corn-vegetable by-product basal diet $+9.95 \%$ meat meal; iii) Poultry meal, cornvegetable by-product basal diet $+9.95 \%$ poultry meal; iv) Tenebrio molitor, corn-vegetable by-product basal diet $+9.95 \%$ dried Tenebrio molitor larvae.

Results: Results showed that the apparent ileal digestibility (AID) of Lys was higher $(\mathrm{p}<0.05)$ in pigs fed Tenebrio molitor diet than that in pigs fed fish meal diet. Pigs fed Tenebrio molitor diet showed increased $(\mathrm{p}<0.05)$ AID of His and Arg compared to pigs fed Fish meal or Meat meal diet. The AID of Cys was increased $(\mathrm{p}<0.05)$ in pigs fed poultry meal and Tenebrio molitor diets compared to that in pigs fish meal diet. Pigs fed meat meal, poultry meal, and Tenebrio molitor diets showed higher $(\mathrm{p}<0.05)$ standardized ileal digestibility (SID) of total energy compared to pigs fed fish meal diet. The SID of Arg was higher $(\mathrm{p}<0.05)$ in pigs fed Tenebrio molitor diet than that in pigs fed fish meal or meat meal diet. Furthermore, pigs fed poultry meal or Tenebrio molitor diets showed increased $(\mathrm{p}<0.05)$ SID of Cys compared to pigs fed fish meal diet.

Conclusion: In conclusion, providing pigs with diets that contained Tenebrio molitor larvae meal improved AID and SID of nutrients as well as essential and non-essential amino acids. The digestibility of dried mealworm larvae protein and its utilization in vivo are also good. Therefore, dried mealworm larvae protein can be used as protein source at $10 \%$ level in growing pigs.

Keywords: Tenebrio molitor; Ileal Amino Acid Digestibility; Growing Pig

\section{INTRODUCTION}

The world population is expected to reach 9 billion in 2050 and production demand of meat and milk are expected to be 58\% and 70\% higher in 2050 than their levels in 2010 [1,2]. Increasing global population and meat consumption have led to urgent need for additional supplies of protein to be included in livestock feed. However, world's agricultural land area is about 5 billion hectares, which is about $37 \%$ of the planet's area. The area suitable for the production of crops, feed, and food is only 1.35 billion hectares. There is a limit to increase 
the production of crops to meet the increasing requirement of vegetable protein raw materials (soybean meal etc.) for livestock. Marine overexploitation has reduced the abundance of small pelagic forage fish from which fish meal and fish oil are derived for aquaculture feed. Prices of soybean meal and fish meal as traditional protein feed stocks will rise sharply which will limit their usage in the future.

To have sustainable protein-rich sources for aquaculture and livestock, one approach is to use insects as protein source. Both the scientific community and food and feed industrial sectors have begun to reconsider the use of insects as an alternative protein source as insects can be efficiently grown on organic side stream [3]. Such researches have so far focused on five major species or species groups $[1,4,5]$ : the common housefly (Musca domestica), the black soldier fly (Hermetia illucens), the yellow mealworm (Tenebrio molitor), locusts (Locusta migratoria, Schistocerca gregaria, Oxya spec., etc.) and silkworms (Bombyx mori, etc.).

Insects are rich in proteins, fats, minerals, and vitamins. Their protein has a higher utilization rate than proteins of other animal by-products, thus attracting attention as a substitute for plant protein source $[6,7]$. In general, proteins contents in insects are $40 \%$ to $65 \%$, similar to protein contents of meat meals. Lipid contents in insects (up to $36 \%$ oil) are also similar to lipid contents of meat meals. Unsaturated fatty acid concentration varies according to insect species: $60 \%$ to $70 \%$ in housefly maggot meal, yellow mealworm, and house cricket; $19 \%$ to $37 \%$ in black soldier fly larvae [4]. It is characterized by high lauric acid (C12:0) content, especially in black soldier fly larvae (Hermetia illucens). Some of them contain large amounts of zinc and iron [8]. However, most insect meals are deficient in Ca. In addition, insects can accumulate or secrete antimicrobial peptides in their body as a defense mechanism against pathogenic microorganisms [9]. It is believed that insects contain chitin which can enhance the immune function of livestock [10]. Makkar et al [4] have confirmed that palatability of insect larvae meal or insect meal to animals is good. Thus, insect larvae meal or insect meal could be used to replace $25 \%$ to $100 \%$ of soymeal or fishmeal depending on animal species.

Dried mealworm larvae contain high amounts of crude protein (CP, $46 \%$ to $52 \%)$ and fat (25\% to $35 \%)$ which contain abundant essential fatty acids with superior oxidative stability [11]. Fresh larvae contain about 60\% water. They have low $\mathrm{Ca}$ content and very low $\mathrm{Ca}: \mathrm{P}$ ratio as other insects. Their $\mathrm{Ca}$ is slightly less available than Ca from oyster shells [12]. Exclusive feeding of mealworms can lead to Ca deficiency and symptomatic metabolic bone disease [12] because of their low $\mathrm{Ca}$ content and low Ca:P ratio. Studies on the feed value of the mealworm larvae have been done in poultry [4,13-17] and swine $[18,19]$, but the results of the study were not enough.

Although information about the effect of mealworm feeding is insufficient, previous studies have indicated that insect larvae powder is a potentially promising protein source. Therefore, the objective of this study was to investigate the nutrient ileal digestibility of dried mealworm larvae in growing pigs compared to three animal protein by-products.

\section{MATERIALS AND METHODS}

\section{Experimental animal and design}

A total of 12 crossbred ([Landrace $\times$ Yorkshire $] \times$ Duroc) growing pigs with initial average body weight of $24.12 \pm 0.68 \mathrm{~kg}$ were surgically equipped with simple T-cannulas after being deprived of feed for $24 \mathrm{~h}$ according to surgical procedures of Stein et al [20]. These pigs had a recovery period of two weeks. A total of 12 pigs were assigned to individual metabolic crates and allotted to one of four treatments with 3 replicates in a fully randomized design. Dietary treatments included the following: i) Fish meal, corn-vegetable by-product basal diet+9.95\% fish meal (TripleNine, Esbjerg, Denmark); ii) Meat meal, cornvegetable by-product basal diet+9.95\% meat meal (DK O\&T, Eumseong, Korea); iii) Poultry meal, corn-vegetable by-product basal diet+9.95\% poultry meal (Harim Co, Ltd., Iksan, Korea); and iv) Tenebrio molitor, corn-vegetable by-product basal diet+ 9.95\% Tenebrio molitor (mealworm) larvae (3.5-month old, Daehan Feed Co, Ltd., Incheon, Korea). Three animal protein sources are commonly used in the livestock feed industry and the mealworms were harvested until about 3.5-month of age and obtained from Daehan feed's insect experimental farm (Yesan, Korea). Tenebrio molitor (mealworm) larvae were dried by a hot-air drier (KEIL-2000, Keil, Seoul, Korea) and ground wholly by grinder for mealworm powder type. Room temperature was maintained at $27^{\circ} \mathrm{C}$.

Chemical constituents of fish meal, meat meal, poultry meal, and dried mealworm (Tenebrio molitor) larvae are shown in Table 1 .

\section{Experimental diet and feeding}

Corn-vegetable by-product-based experimental diets were formulated to contain fish meal, meat meal, poultry meal, and dried mealworm (Tenebrio molitor) larvae powder at level of $9.95 \%$, respectively. Chromic oxide was mixed to the diet at $0.5 \%$ as an indigestible marker to calculate digestibility. Metabolizable energy, $\mathrm{CP}$, methionine, lysine, $\mathrm{Ca}$, and $\mathrm{P}$ requirement of experimental diets were adjusted according to requirements of NRC [21,22]. Raw materials, chemical composition, and amino acid (AA) composition of experimental diets are shown in Table 2 and 3. Raw materials and chemical composition of nitrogen-free diets are shown in Table 4. For N-free diets, protein level was adjusted to the minimum with tapioca starch as base. Each pig was fed $1 \mathrm{~kg}$ of experimental diet four times a day at 07:00, 11:00, 15:00, and 19:00 h which was 2.8 times the maintenance energy requirement $\left(\mathrm{MEn}=106 \mathrm{kcal} \mathrm{ME} / \mathrm{kg}^{0.75}\right.$ [21]). Pigs were provided free access to water at any time via 
Table 1. Composition of fish meal, meat meal, poultry meal and dried mealworm larvae

\begin{tabular}{|c|c|c|c|c|}
\hline $\begin{array}{l}\text { Chemical } \\
\text { composition (\%) }\end{array}$ & $\begin{array}{l}\text { Fish } \\
\text { meal }\end{array}$ & $\begin{array}{l}\text { Meat } \\
\text { meal }\end{array}$ & $\begin{array}{c}\text { Poultry } \\
\text { meal }\end{array}$ & $\begin{array}{c}\text { Dried mealworm } \\
\text { larvae }\end{array}$ \\
\hline Moisture & 8.0 & 4.0 & 4.0 & 5.9 \\
\hline Crude protein & 69.0 & 73.0 & 65.5 & 47.8 \\
\hline Crude fat & 8.5 & 10.0 & 13.0 & 34.6 \\
\hline Crude fiber & 0.8 & 0.5 & 2.0 & 6.1 \\
\hline Crude ash & 15.0 & 15.0 & 12.8 & 6.3 \\
\hline $\mathrm{Ca}$ & 3.20 & 5.00 & 3.40 & 0.05 \\
\hline Total P & 2.30 & 2.40 & 2.10 & 0.59 \\
\hline \multicolumn{5}{|l|}{ Amino acid $^{2)}$} \\
\hline Essential & 29.19 & 21.31 & 24.55 & 42.30 \\
\hline Methionine & 1.86 & 1.00 & 1.38 & 1.34 \\
\hline Cystine & 0.62 & 0.44 & 0.59 & 0.86 \\
\hline Valine & 3.31 & 2.70 & 2.95 & 4.98 \\
\hline Isoleucine & 2.76 & 1.83 & 2.36 & 3.76 \\
\hline Leucine & 5.04 & 3.80 & 4.45 & 6.88 \\
\hline Phenylalanine & 2.70 & 2.20 & 2.42 & 3.72 \\
\hline Tyrosine & 2.20 & 1.61 & 2.09 & 7.76 \\
\hline Histidine & 2.07 & 1.31 & 1.38 & 2.93 \\
\hline Lysine & 5.18 & 3.65 & 3.86 & 5.42 \\
\hline Threonine & 2.69 & 2.26 & 2.55 & 4.04 \\
\hline Tryptophan & 0.76 & 0.51 & 0.52 & 0.61 \\
\hline Non-essential & 32.79 & 43.74 & 35.70 & 48.65 \\
\hline Serine & 2.69 & 2.63 & 2.55 & 4.83 \\
\hline Arginine & 3.80 & 5.04 & 4.39 & 5.46 \\
\hline Glutamic acid & 8.63 & 8.30 & 8.45 & 11.54 \\
\hline Aspartic acid & 6.42 & 5.69 & 4.30 & 8.01 \\
\hline Proline & 3.04 & 6.24 & 5.65 & 6.73 \\
\hline Glycine & 4.00 & 10.51 & 6.03 & 5.06 \\
\hline Alanine & 4.21 & 5.33 & 4.52 & 7.02 \\
\hline
\end{tabular}

1) Lab. of Daehanfeed Co. LTD.

2) $\mathrm{g} / 16 \mathrm{~g}$ nitrogen.

a drinking nipple.

\section{Sample collection}

Samples of ileal digested content were collected between 8:00 and 20:00 $\mathrm{h}$ for three days after five days of adaptation. Ileal digesta were collected into plastic bags attached to the cannulas and were emptied into a plastic containers containing ice every 20 minutes. All samples were immediately transferred after sampling and stored in a $-60^{\circ} \mathrm{C}$ deep freezer to prevent changes in AA sequence due to microbes until analysis. Samples were dried in a freezing dryer and finely ground to pass through a 1-mm screen for chemical analysis including moisture, $\mathrm{CP}$, and AAs contents.

\section{Chemical analysis}

Diets and collected samples were grounded by a Cyclotec CT 193 Sample Mill (Foss Tecator, Hillerod, Denmark) and then analyzed. Analysis of the dry matter (DM) was conducted according to AOAC Method 967.03 [23]. The nitrogen content
Table 2. Composition of the experimental diets, as-fed basis

\begin{tabular}{|c|c|c|c|c|}
\hline \multirow[b]{2}{*}{ Items } & \multicolumn{4}{|c|}{ Treatments } \\
\hline & Fish meal & $\begin{array}{l}\text { Meat } \\
\text { meal }\end{array}$ & $\begin{array}{c}\text { Poultry } \\
\text { meal }\end{array}$ & $\begin{array}{c}\text { Tenebrio } \\
\text { molitor }\end{array}$ \\
\hline \multicolumn{5}{|l|}{ Ingredients (\%) } \\
\hline Ground corn & 73.89 & 73.59 & 75.89 & 63.27 \\
\hline Soybean meal, 45\% & 5.14 & 4.69 & 5.65 & 9.35 \\
\hline Fish meal & 9.95 & 0 & 0 & 0 \\
\hline Meat meal & 0 & 9.95 & 0 & 0 \\
\hline Poultry meal & 0 & 0 & 9.95 & 0 \\
\hline Meal worm & 0 & 0 & 0 & 9.95 \\
\hline Palm kernel meal & 2.98 & 3.00 & 2.99 & 2.99 \\
\hline Wheat bran & 4.64 & 6.14 & 1.90 & 10.42 \\
\hline Tallow & 1.16 & 1.17 & 1.17 & 0.67 \\
\hline MDCP & 0.48 & 0 & 0.53 & 0.94 \\
\hline Limestone & 0.66 & 0.03 & 0.61 & 1.07 \\
\hline DL-methionine, 99\% & 0 & 0.09 & 0.05 & 0.10 \\
\hline L-lysine-HCl, 78\% & 0.13 & 0.34 & 0.27 & 0.24 \\
\hline Salt & 0.30 & 0.30 & 0.30 & 0.30 \\
\hline Vitamin premix ${ }^{11}$ & 0.10 & 0.10 & 0.10 & 0.10 \\
\hline Mineral premix ${ }^{2)}$ & 0.10 & 0.10 & 0.10 & 0.10 \\
\hline $\mathrm{Cr}_{2} \mathrm{O}_{3}$ & 0.50 & 0.50 & 0.50 & 0.50 \\
\hline Sum & 100 & 100 & 100 & 100 \\
\hline \multicolumn{5}{|l|}{ Chemical composition ${ }^{3)}$} \\
\hline ME (kcal/kg) & 3,249 & 3,249 & 3,249 & 3,249 \\
\hline Crude protein (\%) & 15.50 & 15.50 & 15.50 & 15.50 \\
\hline Crude fat (\%) & 4.52 & 4.71 & 4.97 & 6.01 \\
\hline Crude fiber (\%) & 2.61 & 2.67 & 2.37 & 3.70 \\
\hline Crude ash (\%) & 5.21 & 4.16 & 4.93 & 5.62 \\
\hline Lysine (\%) & 0.95 & 0.95 & 0.95 & 0.95 \\
\hline Methionine (\%) & 0.35 & 0.35 & 0.35 & 0.35 \\
\hline $\mathrm{Ca}(\%)$ & 0.66 & 0.66 & 0.66 & 0.66 \\
\hline Total P (\%) & 0.56 & 0.58 & 0.56 & 0.56 \\
\hline
\end{tabular}

MDCP, mono-di-calcium phosphate (Ca, $17.6 \%$ and total $P, 20.4 \%)$.

1) Provided the following per kilogram of diet: vitamin $A, 7,960 \mathrm{IU}$ as vitamin $A$ acetate; vitamin $\mathrm{D}_{31}, 1,592 \mathrm{IU}$; vitamin $\mathrm{E}, 32 \mathrm{IU}$ as dl- $\alpha$-tocopheryl acetate; biotin, $0.25 \mathrm{mg}$ as d-biotin; riboflavin, $3.2 \mathrm{mg}$; thiamine $3 \mathrm{mg}$ as thiamine mononitrate; pyridoxine $5 \mathrm{mg}$ as pyridoxine hydrochloride; folic acid $0.5 \mathrm{mg}$; pantothenic acid, 8 $\mathrm{mg}$ as d-calcium pantothenate; niacin, $46 \mathrm{mg}$ as nicotinic acid; vitamin $\mathrm{B}_{121} 12 \mu \mathrm{g}$ as cyanocobalamin; and vitamin $\mathrm{K}_{3}, 2.4 \mathrm{mg}$ as menadione nicotinamide bisulfate.

2) Provided the following per kilogram of diet: $\mathrm{Cu} 24.8 \mathrm{mg}$ as $\mathrm{CuSO}_{4} \mathrm{H}_{2} \mathrm{O} ; \mathrm{Fe} 54.1$ mg as $\mathrm{FeSO}_{4} \mathrm{H}_{2} \mathrm{O} ; \mathrm{Zn} 84.7 \mathrm{mg}$ as $\mathrm{ZnSO}_{4} \mathrm{H}_{2} \mathrm{O} ; \mathrm{Mn} 24.8 \mathrm{mg}$ as $\mathrm{MnSO}_{4} \mathrm{H}_{2} \mathrm{O} ; \mathrm{I} 0.3 \mathrm{mg}$ as $\mathrm{Ca}\left(\mathrm{IO}_{3}\right) \mathrm{H}_{2} \mathrm{O} ; \mathrm{Co} 0.3 \mathrm{mg}$ as $\mathrm{CoSO}_{4} \mathrm{H}_{2} \mathrm{O}$; and $\mathrm{Se} 0.1 \mathrm{mg}$ as $\mathrm{NaSeO}_{3} \mathrm{H}_{2} \mathrm{O}$.

${ }^{3)}$ Calculated values.

was analyzed by using the Kjeldahl procedure with Kjeltec (Kjeltec TM2200, Foss Tecator, Höganäs, Sweden) and calculating the CP content (Nitrogen $\times 6.25$; procedure 981.10; AOAC [23]). For the analysis of AA except for methionine and cysteine, diets and samples were hydrolyzed in $6 \mathrm{~N} \mathrm{HCl}$ at $110^{\circ} \mathrm{C}$ for 24 hours (AOAC Method 999.13 [23]). Methionine and cystine were determined after cold performic acid oxidation overnight and hydrolyzed with $7.5 \mathrm{~N} \mathrm{HCl}$ (AOAC method 994.12 [23]). Individual AA was measured by using an AA analyzer (Beckman 6300 Amino Acid Analyzer; Beckman Instruments Corp., Palo Alto. CA, USA). Chromium 
Table 3. Amino acid composition of experimental diets

\begin{tabular}{lrrrrr}
\hline & \multicolumn{5}{c}{ Treatments } \\
\cline { 2 - 5 } Items & $\begin{array}{c}\text { Fish } \\
\text { meal }\end{array}$ & $\begin{array}{c}\text { Meat } \\
\text { meal }\end{array}$ & $\begin{array}{c}\text { Poultry } \\
\text { meal }\end{array}$ & $\begin{array}{c}\text { Tenebrio } \\
\text { molitor }\end{array}$ & \\
\hline Total amino acid (\%) & SEM \\
Essential amino acid $(\%)^{3)}$ & 15.07 & 13.40 & 15.19 & 16.09 & 0.971 \\
Lys & 0.98 & 0.88 & 1.10 & 1.21 & 0.124 \\
Met & 0.28 & 0.28 & 0.23 & 0.29 & 0.023 \\
Thr & 0.71 & 0.66 & 0.72 & 0.76 & 0.036 \\
Val & 0.60 & 0.57 & 0.69 & 0.73 & 0.065 \\
Ile & 0.56 & 0.54 & 0.67 & 0.71 & 0.072 \\
Leu & 1.22 & 1.10 & 1.24 & 1.32 & 0.079 \\
Phe & 0.75 & 0.64 & 0.74 & 0.79 & 0.055 \\
His & 0.38 & 0.34 & 0.39 & 0.41 & 0.025 \\
Arg & 1.03 & 0.88 & 1.04 & 1.10 & 0.081 \\
Non-essential amino acid $(\%)^{3)}$ & & & & \\
Asp & 1.66 & 1.41 & 1.63 & 1.68 & 0.108 \\
Ser & 0.83 & 0.71 & 0.80 & 0.83 & 0.049 \\
Glu & 2.75 & 2.38 & 2.71 & 2.87 & 0.182 \\
Gly & 0.77 & 0.67 & 0.74 & 0.78 & 0.043 \\
Ala & 0.83 & 0.76 & 0.81 & 0.85 & 0.033 \\
Tyr & 0.44 & 0.37 & 0.44 & 0.48 & 0.040 \\
Pro & 1.04 & 0.97 & 1.03 & 1.05 & 0.031 \\
Cys & 0.24 & 0.24 & 0.21 & 0.23 & 0.012 \\
\hline SEM & & & & &
\end{tabular}

SEM, standard error of means.

1) Fish meal, corn-vegetable by-product based diet with $9.95 \%$ fish meal; meat meal, corn-vegetable by-product based diet with $9.95 \%$ meat meal; poultry meal, corn-vegetable by-product based diet with $9.95 \%$ poultry meal; Tenebrio molitor, corn-vegetable by-product based diet with $9.95 \%$ dried Tenebrio molitor larvae.

${ }^{2)}$ Total amino acid: Essential amino acid (\%)+non-essential amino acid (\%).

${ }^{3)}$ Chemical analysis values.

concentrations were determined via UV absorption spectrophotometry (UV-1201, Shimadzu, Kyoto, Japan). Gross energy was determined using a Parr 6100 oxygen bomb calorimeter (Parr instrument Co., Moline, IL, USA).

\section{Calculations}

Standardized ileal digestibility (SID) of AA was calculated according to the method described by Stein $[24,25]$ as an objective indicator of AA digestibility. Basal endogenous losses of CP and AAs were measured using samples collected at the end of the ileum after feeding with non-nitrogen feed [26]. Calculation of digestibility was done based on the relative chromium concentration of feed and ileal samples. AID and SID were calculated using the following equations:

$$
\begin{aligned}
& \text { Apparent ileal digestibility (AID, \%) } \\
& =100-[(\mathrm{ND} / \mathrm{NF}) \times(\mathrm{CrF} / \mathrm{CrD}) \times 100]
\end{aligned}
$$

Basal endogenous AA losses $(\mathrm{EAL})=[\mathrm{ND} \times(\mathrm{CrF} / \mathrm{CrD})]$

$$
\operatorname{SID}(\%)=[\mathrm{AID}+(\mathrm{EAL} / \mathrm{NF})] \times 100
$$

\begin{tabular}{|c|c|}
\hline Items & \\
\hline \multicolumn{2}{|l|}{ Ingredient (\%) } \\
\hline Tapioca starch & 67.25 \\
\hline Soy-oil & 9.22 \\
\hline Sucrose & 9.95 \\
\hline Lactose & 9.95 \\
\hline MDCP & 2.63 \\
\hline Limestone & 0.00 \\
\hline Salt & 0.30 \\
\hline Vitamin premix ${ }^{1)}$ & 0.10 \\
\hline Mineral premix & 0.10 \\
\hline $\mathrm{Cr}_{2} \mathrm{O}_{3}$ & 0.50 \\
\hline Sum & 100.0 \\
\hline \multicolumn{2}{|l|}{ (hemical composition) } \\
\hline ME (kcal/kg) & 3,249 \\
\hline Crude protein (\%) & 0.00 \\
\hline Lysine (\%) & 0.00 \\
\hline Methionine (\%) & 0.00 \\
\hline Threonine (\%) & 0.00 \\
\hline $\mathrm{Ca}(\%)$ & 0.70 \\
\hline Total P (\%) & 0.60 \\
\hline \multicolumn{2}{|c|}{ 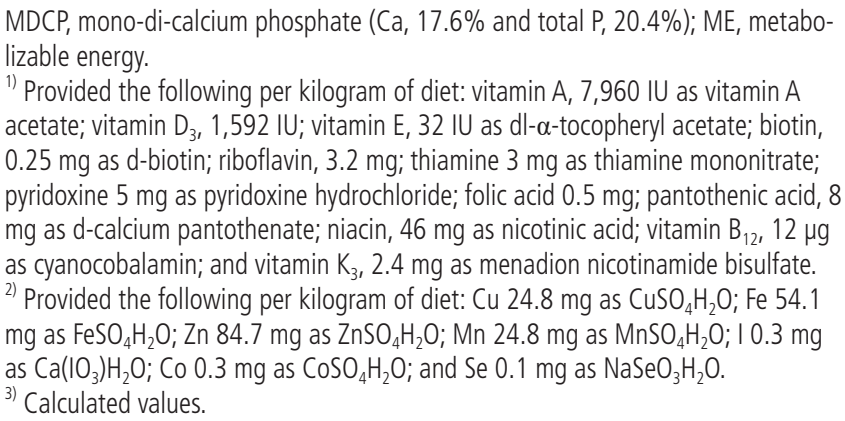 } \\
\hline
\end{tabular}

Table 4. Composition of the nitrogen-free diets, as-fed basis

Where, ND, NF, CrF, and CrD were nutrient concentration in the ileum sample, nutrient concentration in the feed, chromium concentration in the feed, and chromium concentration in the ileum sample, respectively.

\section{Statistical analysis}

Each pig was considered as experimental unit. All data were analyzed using the general linear model procedure of SAS (SAS Inst. Inc., Cary, NC, USA). Results were compared by the least significant difference multiple test method. Differences among treatment means were determined using Tukey's test with significance level at $\mathrm{p}<0.05$.

\section{RESULTS}

Nutrient contents of dried mealworm larvae and the other three animal protein sources are summarized in Table 1. These data indicated that mealworm larvae contained higher percentage of essential AAs (46.3\%) than other protein sources. Results of AID are presented in Table 5. The AID of Lys was 
Table 5. Effect of protein source on apparent ileal digestibility in growing pigs

\begin{tabular}{|c|c|c|c|c|c|c|}
\hline \multirow[b]{2}{*}{ Items } & \multicolumn{4}{|c|}{ Treatments ${ }^{1)}$} & \multirow[b]{2}{*}{ SEM } & \multirow[b]{2}{*}{ p-value } \\
\hline & $\begin{array}{l}\text { Fish } \\
\text { meal }\end{array}$ & $\begin{array}{l}\text { Meat } \\
\text { meal }\end{array}$ & $\begin{array}{c}\text { Poultry } \\
\text { meal }\end{array}$ & $\begin{array}{l}\text { Tenebrio } \\
\text { molitor }\end{array}$ & & \\
\hline Dry matter (\%) & 84.35 & 86.99 & 87.65 & 89.44 & 2.679 & 0.08 \\
\hline Gross energy (\%) & 83.62 & 88.39 & 87.86 & 89.53 & 3.135 & 0.06 \\
\hline Crude protein (\%) & 85.04 & 86.76 & 87.87 & 89.58 & 2.387 & 0.05 \\
\hline Total amino acid (\%) & 85.37 & 86.90 & 87.97 & 89.60 & 1.543 & 0.05 \\
\hline \multicolumn{7}{|c|}{ Essential amino acid (\%) } \\
\hline Lys & $86.13^{b}$ & $87.36^{\mathrm{ab}}$ & $88.40^{\mathrm{ab}}$ & $89.65^{\mathrm{a}}$ & 1.296 & 0.04 \\
\hline Met & 85.53 & 87.19 & 87.76 & 89.56 & 1.440 & 0.06 \\
\hline Thr & 84.17 & 86.82 & 87.59 & 89.53 & 1.925 & 0.06 \\
\hline Val & 84.34 & 85.99 & 87.88 & 89.51 & 1.946 & 0.05 \\
\hline lle & 84.07 & 86.25 & 87.87 & 89.38 & 1.971 & 0.05 \\
\hline Leu & 85.05 & 86.41 & 87.89 & 89.59 & 1.691 & 0.05 \\
\hline Phe & 85.66 & 86.88 & 87.98 & 89.59 & 1.448 & 0.06 \\
\hline His & $85.51^{b}$ & $86.68^{b}$ & $88.28^{\mathrm{ab}}$ & $89.68^{a}$ & 1.579 & 0.04 \\
\hline $\operatorname{Arg}$ & $87.37^{\mathrm{b}}$ & $87.66^{b}$ & $88.93^{\mathrm{ab}}$ & $89.74^{\mathrm{a}}$ & 0.961 & 0.03 \\
\hline \multicolumn{7}{|c|}{ Non-essential amino acid (\%) } \\
\hline Asp & 85.66 & 87.10 & 87.96 & 89.60 & 1.427 & 0.05 \\
\hline Ser & 85.14 & 87.03 & 88.04 & 89.61 & 1.622 & 0.06 \\
\hline Glu & 86.44 & 87.47 & 88.34 & 89.72 & 1.202 & 0.06 \\
\hline Gly & 84.22 & 86.39 & 87.14 & 89.53 & 1.896 & 0.06 \\
\hline Ala & 83.64 & 86.23 & 86.9 & 89.45 & 2.066 & 0.06 \\
\hline Tyr & 84.45 & 86.57 & 87.5 & 89.47 & 1.806 & 0.06 \\
\hline Pro & 84.97 & 86.76 & 87.64 & 89.62 & 1.676 & 0.07 \\
\hline Cys & $83.00^{\mathrm{b}}$ & $86.02^{\mathrm{ab}}$ & $87.64^{\mathrm{a}}$ & $89.57^{\mathrm{a}}$ & 2.406 & 0.04 \\
\hline
\end{tabular}

SEM, standard error of means.

1) Fish meal, corn-vegetable by-product based diet with $9.95 \%$ fish meal; meat meal, corn-vegetable by-product based diet with $9.95 \%$ meat meal; poultry meal, corn-vegetable by-product based diet with $9.95 \%$ poultry meal; Tenebrio molitor, corn-vegetable by-product based diet with 9.95\% dried Tenebrio molitor larvae.

${ }^{a b}$ Means in a same row with different superscript significantly different $(p<0.05)$.

higher $(\mathrm{p}<0.05)$ in pigs fed Tenebrio molitor diet compared to that in pigs fed Fish meal diet. Pigs fed Tenebrio molitor diet showed increased $(\mathrm{p}<0.05)$ AID of His and Arg compared to pigs fed Fish meal or Meat meal diet. In addition, the AID of Cys was increased $(\mathrm{p}<0.05)$ in pigs fed Poultry meal or Tenebrio molitor diet compared to pigs fed Fish meal diet. Although there were no significant differences, pigs fed Tenebrio molitor diet tended to show increased AID of DM, total energy, CP, total AAs, and the rest of essential AAs (Met, Thr, Val, Ile, Leu, and Phe) and non-essential AAs (Asp, Ser, Glu, Gly, Ala, Tyr, and Pro) than pigs fed fish meal, meat meal, or poultry meal diet. The digestibility of each nutrient showed similar tendency in the case of poultry meat and meat meal diets.

Pigs fed meat meal, poultry meal, or Tenebrio molitor diet had higher $(\mathrm{p}<0.05)$ SID of total energy compared to pigs fed fish meal diet (Table 6). The SID of Arg was higher $(\mathrm{p}<0.05)$ in pigs fed Tenebrio molitor diet compared to that in pigs fed fish meal or meat meal diets. Furthermore, pigs fed poultry meal, meat meal, or Tenebrio molitor diet showed increased $(\mathrm{p}<0.05)$ SID of Cys compared to pigs fed fish meal diet. Pigs
Table 6. Effect of protein source on standardized ileal digestibility in growing pigs

\begin{tabular}{lcccccc}
\hline & \multicolumn{5}{c}{ Treatments $^{\text {1) }}$} & \\
Items & $\begin{array}{c}\text { Fish } \\
\text { meal }\end{array}$ & $\begin{array}{c}\text { Meat } \\
\text { meal }\end{array}$ & $\begin{array}{c}\text { Poultry } \\
\text { meal }\end{array}$ & $\begin{array}{c}\text { Tenebrio } \\
\text { molitor }\end{array}$ & & \\
& SEM & p-value \\
\hline Dry matter (\%) & 85.66 & 88.31 & 88.96 & 89.53 & 2.484 & 0.08 \\
Gross energy (\%) & $83.30^{\mathrm{b}}$ & $90.01^{\mathrm{a}}$ & $88.76^{\mathrm{a}}$ & $90.96^{\mathrm{a}}$ & 3.339 & $<0.01$ \\
Crude protein (\%) & 85.55 & 87.32 & 88.38 & 90.05 & 2.270 & 0.06 \\
Total amino acid (\%) & 85.85 & 87.44 & 88.44 & 90.05 & 1.527 & 0.06 \\
Essential amino acid (\%) & & & & & \\
Lys & 86.51 & 87.78 & 88.73 & 89.96 & 1.264 & 0.05 \\
Met & 85.87 & 87.53 & 88.17 & 89.89 & 1.439 & 0.06 \\
Thr & 84.79 & 87.50 & 88.21 & 90.12 & 1.910 & 0.06 \\
Val & 85.02 & 86.71 & 88.47 & 90.07 & 1.891 & 0.06 \\
Ile & 84.77 & 86.98 & 88.46 & 89.93 & 1.908 & 0.06 \\
Leu & 85.45 & 86.85 & 88.28 & 89.96 & 1.675 & 0.06 \\
Phe & 86.01 & 87.29 & 88.34 & 89.93 & 1.436 & 0.06 \\
His & 85.94 & 87.15 & 88.69 & 90.07 & 1.561 & 0.05 \\
Arg & $87.61^{\mathrm{b}}$ & $87.95^{\mathrm{b}}$ & $89.17^{\mathrm{ab}}$ & $89.97^{\mathrm{a}}$ & 0.947 & 0.04 \\
Non-essential amino acid (\%) & & & & & \\
Asp & 86.06 & 87.57 & 88.37 & 90.00 & 1.420 & 0.06 \\
Ser & 85.66 & 87.64 & 88.58 & 90.13 & 1.619 & 0.06 \\
Glu & 86.75 & 87.83 & 88.65 & 90.01 & 1.193 & 0.06 \\
Gly & 84.82 & 87.08 & 87.76 & 90.12 & 1.890 & 0.07 \\
Ala & 84.17 & 86.8 & 87.43 & 89.96 & 2.060 & 0.07 \\
Tyr & 84.84 & 87.03 & 87.89 & 89.83 & 1.791 & 0.06 \\
Pro & 86.08 & 87.96 & 88.77 & 90.73 & 1.667 & 0.07 \\
Cys & $83.62^{\mathrm{b}}$ & $86.64^{\mathrm{ab}}$ & $88.35^{\mathrm{a}}$ & $90.21^{\mathrm{a}}$ & 2.425 & 0.04 \\
\hline
\end{tabular}

SEM, standard error of means.

1) Fish meal, corn-vegetable by-product based diet with $9.95 \%$ fish meal; meat meal, corn-vegetable by-product based diet with $9.95 \%$ meat meal; poultry meal, corn-vegetable by-product based diet with $9.95 \%$ poultry meal; Tenebrio molitor, corn-vegetable by-product based diet with 10\% dried Tenebrio molitor larvae.

${ }^{a b}$ Means in a same row with different superscript significantly different $(p<0.05)$.

fed with Tenebrio molitor diet tended to show increased SID of DM, total energy, CP, total AAs, the rest of essential AAs (Lys, Met, Thr, Val, Ile, Leu, Phe, and His), and the rest of nonessential AAs (Asp, Ser, Glu, Gly, Ala, Tyr, and Pro) than pigs fed meat meal, poultry meal, or fish meal diet.

\section{DISCUSSION}

In general, dried mealworm larvae contain high amounts of CP (46\% to $52 \%)$ and fat (25\% to $35 \%)$ with relatively low ash content $[4,27]$. Fresh larvae contain about $60 \%$ moisture. They are also relatively low in Ca content. The dried mealworm larvae used in this study were about 3.5-month old with CP, essential AA, crude fat, and Ca contents of $48.2 \%, 46.3(\mathrm{~g} / 16 \mathrm{~g}-\mathrm{N})$, $29.5 \%$, and $0.04 \%$, respectively. The AA profiles and protein contents of dried mealworm larvae and three animal protein by-products are superior to soybean meal, the main protein source used in pig and poultry feed. Soybean meal has a CP content of $49 \%$ to $56 \% \mathrm{DM}$ and has a crude fat content of $3 \%$ 
DM [28]. The other three animal protein sources have protein content of $65.5 \%$ to $73 \%$ in as-fed basis. With regard to protein content and AA quality, dried mealworm larvae are comparable to meat meal and poultry meal as well as fish meal. The other three animal protein sources have protein content of $65.5 \%$ to $73 \%$ in as-fed basis. Essential AA (lysine, methionine+cystine, threonine and tryptophan) index, EAAI [29] values for fish meal, meat meal, poultry meal and dried mealworm larvae were $0.57,0.40,0.45$, and 0.58 , respectively (AA requirements based on Daehan feed Co. data). EAAI of dried mealworm larvae was similar to that of fish meal.

Fat content of animal protein source in this experiment was 9.71\% for fish meal, $11.09 \%$ for meat meal, $13.00 \%$ for poultry meal, and $29.5 \%$ for dried mealworm (Tenebrio molitor) larvae. Because experimental diets were formulated with the same energy and dried mealworm (Tenebrio molitor) larvae had the highest fat content and the lowest ash content, Tenebrio molitor diet had lower ground corn content and higher wheat bran content resulting in higher crude fiber content than the other three diets. This might can have an impact when designing grower feed formula and feeding the diet to grower. Although excessively high fiber content of diet limits feed intake, adequate fiber content can have a beneficial effect on the environment of large intestine. Previously studies have measured CP digestibility of some insect larvae [19,30-32]. Newton et al [31] have reported that the apparent faecal digestibility of black soldier fly larvae in male growing pigs is $76 \%$ similar apparent CP digestibility as soybean meal. Jin et al [19] have shown that the total track digestibility of $\mathrm{CP}$ is linearly increased with increasing dried mealworm level. The digestibility in their studies was 92.17\% (level of dried mealworm larvae included in the diet was $4.5 \%$ ) or $93.04 \%$ (level of dried mealworm larvae included in the diet was 6.0\%). That of our experiment is higher than that of Newton et al [31] and lower than that of Jin et al [19]. In general, the apparent total track protein digestibility is lower than the ileal digestibility due to microorganisms in the large intestine. In addition, the age of the experimental animals [31] was 5 weeks at which the development of protein digestion enzymes was not sufficient. Although the protein digestibility of Jin et al [19] was a total track digestibility and the pigs were younger than those of this study, that was higher than that of this experiment due to experimental diet composition (mainly soybean meal was used as vegetable protein by-product) expected to have a higher digestibility than palm kernel meal and wheat bran, and lower feeding amount ( $2 \%$ of body weight) than that of this study ( $4 \%$ of body weight).

The protein digestibility of the dried mealworm larvae containing diets tended to be slightly higher than those of the other experimental diets containing other animal by-products $(\mathrm{p}=$ 0.05 ). Increased dietary protein digestibility aids weight gain in chicks [30] and weaning pigs [19]. Although growth performance was not determined in this study, improved growth performance with dried mealworm larvae could be deduced with current result of protein digestibility when compared with other three animal by-products.

Hwangbo et al [30] and Pretorius [32] have conducted studies using housefly meal in broilers and reported digestibility of $98.5 \%$ and $69 \%$, respectively. However, the later study reported that the AA digestibility was over $90 \%$ while the $\mathrm{CP}$ digestibility was much lower. This might have attributed to indigestibility of chitin-N and/or ADF bound-N [32]. Chitin, a linear polymer of $\beta$-(1-4) N-acetyl-D-glucosamine units with a chemical structure similar to that of cellulose, is combined with protein and distributed widely on cell wall and shell of crabs, shrimp, and insects. Chitin is rarely found in nature alone. It is distributed in the form of chitin-protein complexes as a major component of the cuticle layer [33]. In addition, proteins of Tenebrio molitor larvae are mainly composed of proteins derived from cuticle and other proteins, including various hemolymph proteins, endurance proteins (such as hexamerin), and different enzymatic proteins [34]. In this study, the protein digestibility was relatively high (AID, $89.58 \%$; SID, 90.05\%) and there was no significant difference when compared with those of total AA digestibility (AID, 89.60\%; SID, 90.05\%). There was little effect of chitin on protein digestibility. The chitin content of mealworm larvae has been estimated to be $2.8 \%$ of DM [35]. Although dried mealworm larvae have proteinchitin complex, the inclusion rate of that was only $9.95 \%$ in total experimental diet. It had little effect on digestibility of total feed protein. It can be deduced that there is no need to consider protein-chitin complex at about $10 \%$ level as a protein source.

Pigs fed Tenebrio molitor larvae meal showed similar AID of lysine (89.65\%), histidine (89.68\%), and arginine (89.74\%) with results of Ji et al [18], reporting that AID values of lysine, histidine, and arginine were $89.33 \%, 88.00 \%$, and $92.00 \%$, respectively, in early-weaned piglets (Phase 2, Day 29 to 56). Even though the pigs were younger than those of this study, the digestibility was similar due to complexity of experimental diet, having more digestible raw materials. However there was significant variation in AID values of alanine, cystine, and glycine $(78.67 \%, 77.00 \%$, and $70.67 \%$, respectively) in the results of Ji et al [18] while there was no significant variation in digestibility between AAs in this study. They inferred that the lower AID values were due to different AA structure and said that further studies were needed to explore this possibility [18]. Pigs fed Tenebrio molitor larvae meal showed higher AID and SID of lysine (89.65\% and $89.96 \%)$, histidine ( $89.68 \%$ and $90.07 \%$ ), and arginine (89.74\% and $89.9 \%$ ) in comparison with pigs in other treatment groups.

It would appear from the results of this study, that dried mealworm (Tenebrio molitor) larvae would be a suitable ingredient in growing pigs, being especially valuable from the stand-point ileal AA digestibility. High protein and AA diges- 
tibility of dried mealworm larvae may act on the deposition of AAs in pig meat and on the growth performance in growing pigs. Further studies are needed to explore this possibility.

\section{CONCLUSION}

In conclusion, the nutritional value of dried mealworm larvae closely matched with that of fishmeal, making it a potentially attractive alternative protein-rich feed ingredient for livestock feed industry. Dietary supplementation of dried mealworm larvae had higher digestibility in DM, CP, total AAs, essential AAs, and non-essential AAs compared to dietary supplementation with fish meal, meat meal, or poultry meal. Thus, dried mealworm larvae protein can be used as a protein source at $10 \%$ level in growing pigs.

\section{CONFLICT OF INTEREST}

We certify that there is no conflict of interest with any financial organization regarding the material discussed in the manuscript. Cho KH is an employee of Daehan feed Co., Ltd. and Shin DG is an employee of Berry \& Biofood Research Institute.

\section{ACKNOWLEDGMENTS}

This work was supported by Korea Institute of Planning and Evaluation for Technology in Food, Agriculture, Forestry and Fisheries (IPET) through Technology Commercialization Support Program, funded by Ministry of Agriculture, Food and Rural Affairs (MAFRA) (Project No::817025-03).

\section{REFERENCES}

1. Veldkamp T, Bosch G. Insects: a protein-rich feed ingredient in pig and poultry diets. Anim Front 2015;5:45-50.

2. FAO. World Livestock 2011 - Livestock in food security. Food and Agriculture Organization of the United Nations (FAO), Rome, Italy; 2011.

3. van Huis A. Potential of insects as food and feed in assuring food security. Annu Rev Entomol 2013;58:563-83.

4. Makkar HPS, Tran G, Heuze V, Ankers P. State-of-the-art on use of insects as animal feed. Anim Feed Sci Technol 2014;197: 1-33.

5. Stamer A. Insect proteins-a new source for animal feed. EMBO Rep 2015;16:676-80.

6. Aguilar-Miranda ED, Lopez MG, Escamilla-Santana C, et al. Characteristics of maize flour tortilla supplemented with ground Tenebrio molitor larvae. J Agric Food Chem 2002;50:192-5.

7. Hardouin F, Thys E, Joiris V, et al. Mini-livestock breeding with indigenous species in the tropics. Livest Res Rural Dev 2003; 15:Article \#30.

8. De Foliart GR. The human use of insects as food and as animal feed. Bull Entomol Soc Am 1989;35:22-36.

9. Ratcliffe N, Azambuja P, Mello CB. Recent advances in developing insect natural products as potential modern day medicines. Evid Based Complement Alternat Med 2014;2014:Article ID 904958.

10. Khempaka S, Chitsatchapong C, Molee W. Effect of chitin and protein constituents in shrimp head meal on growth performance, nutrient digestibility, intestinal microbial populations, volatile fatty acids, and ammonia production in broilers. J Appl Poult Res 2011;20:1-11.

11.Jeon $\mathrm{YH}$, Son YJ, Kim SH, et al. Physicochemical properties and oxidative stabilities of mealworm (Tenebrio molitor) oils under different roasting conditions. Food Sci Biotechnol 2016; 25:105-10.

12. Klasing KC, Thacker P, Lopez MA, et al. Increasing the calcium content of mealworms (Tenebrio molitor) to improve their nutritional value for bone mineralization of growing chicks. J Zoo Wildl Med 2000;31:512-8.

13. Ramos-Elorduy J, Gonzalez EA, Hernandez AR, et al. Use of Tenebrio molitor (coleoptera: tenebrioniidae) to recycle organic wastes and as feed for broiler chickens. J Econ Entomol 2002; 95:214-21.

14. Hornung B. The importance of mealworm larvae (Tenebrio molitor, L. 1758) as carriers of zearalenone when fed to insectivorous birds and other pet animals. In: The importance of the larvae of the flour beetle (Tenebrio molitor, L. 1758) as a carrier of zearalenone in the feeding of insectivorous birds and other pets; 1991.pp. 81.

15. Schiavone A, De Marco M, Rotolo L, et al. Nutrient digestibility of Hermetia illucens and Tenebrio molitor meal in broiler chickens. Abstract book Conference "Insects to Feed The World". 2014 May 14-17; Wageningen, The Netherlands. pp. 84.

16. Giannone M. A natural supplement made of insect larvae. Rivista di Avicoltura 2003;72:40-1.

17. Wang YC, Chen YT, Li XR, et al. Study on the rearing larvae of Tenebrio iter Linn'e and the effects of its processing and utilizing. Acta Agriculturae Universitatis Henanensis 1996;30:288-92.

18.Ji YJ, Liu NH, Kong XF, et al. Use of insect powder as a source of dietary protein in early-weaned piglets. J Anim Sci 2016;94: 111-6.

19. Jin XH, Heo PS, Hong JS, et al. Supplementation of dried mealworm (Tenebrio molitor larva) on growth performance, nutrient digestibility and blood profiles in weaning pigs. Asian-Australas J Anim Sci 2016;29:979-86.

20.Stein HH, Shipley CF, Easter RA. Technical note: a technique for inserting a T-cannula into the distal ileum of pregnant sows. J Anim Sci 1998;76:1433-6.

21. NRC. Nutrient requirements of swine (10th Ed.). Washington, DC, USA: National Academy Press; 1998.

22. NRC. Nutrient requirements of swine (11th Ed.). Washington, DC, USA: National Academy Press; 2012.

23. AOAC. Official methods of analysis. 17th edn. Association of 
Official Analysis Chemists (AOAC), Arlington, VA, USA: AOAC International; 2003.

24. Stein HH, Aref S, Easter RA. Comparative protein and amino acid digestibilities in growing pigs and sows. J Anim Sci 1999; 77:1169-79.

25. Stein HH, Kim SW, Nielsen TT, et al. Standardized ileal protein and amino acid digestibility by growing pigs and sows. J Anim Sci 2001;79:2113-22.

26. Stein HH, Trottier NL, Bellaver C, et al. The effect of feeding level and physiological status on total flow and amino acid composition of endogenous protein at the distal ileum in swine. J Anim Sci 1999;77:1180-7.

27. Siemianowska E, Kosewska A, Aljewicz M, et al. Larvae of mealworm (Tenebrio molitor L.) as european novel food. Agric Sci 2013;4:287-91.

28. CVB. Chemical compositions and nutritional values of feed materials. CVB Veevoedertabel 2007. Productschap Diervoeder, Den Haag, The Netherlands; 2007.

29. Smith DM. Protein separation and characterization procedures.
In: Nielsen SS, editor, Food analysis. Chapter 15. New York, NY, USA: Springer Verlag; 2010. pp. 263-80.

30. Hwangbo J, Hong EC, Jang A, et al. Utilization of house flymaggots, a feed supplement in the production of broiler chickens. J Environ Biol 2009;30:609-14.

31. Newton GL, Booram CV, Barker RW, et al. Dried Hermetia illucens larvae meal as a supplement for swine. J Anim Sci 1977; 44:395-400.

32. Pretorius Q. The evaluation of larvae of Musca Domestica (common house fly) as protein source for broiler production [Thesis]. Western Cape, South Africa: University of Stellenbosch; 2011.

33. Vincent JFV, Wegst UGK. Design and mechanical properties of insect cuticle. Arthropod Struct Dev 2004;33:187-99.

34. Kar SK, Jansman AJM, Boeren S, et al. Protein, peptide, amino acid composition, and potential functional properties of existing and novel dietary protein sources for monogastrics. J Anim Sci 2016;94:30-9.

35. Finke MD. Complete nutrient content of four species of feeder insects. Zoo Biol 2013;32:27-36. 